\title{
Protein, Organized by Function
}

National Cancer Institute

\section{Source}

National Cancer Institute. Protein, Organized by Function. NCI Thesaurus. Code C20027.

Organizing term for the Proteins tree 